\title{
La adaptabilidad en las Normas Técnicas para Proyectos de Conjuntos Habitacionales IVBA: un análisis desde la Investigación Proyectual.
}

Fiscarelli*, Diego

La adaptabilidad en las Normas Técnicas para Proyectos de Conjuntos Habitacionales IVBA: un análisis desde la Investigación Proyectual.

Estudios del Hábitat, vol. 18, núm. 1, 2020

Universidad Nacional de La Plata, Argentina

Atribución no comercial compartir igual (CC BY-NC-SA) 4.0 


\title{
La adaptabilidad en las Normas Técnicas para Proyectos de Conjuntos Habitacionales IVBA: un análisis desde la Investigación Proyectual.
}

\author{
Diego Fiscarelli* \\ Laboratorio de Tecnología y Gestión Habitacional. \\ Facultad de Arquitectura y Urbanismo. Universidad \\ Nacional de La Plata, Argentina \\ dfiscarelli@fau.unlp.edu.ar
}

Adaptability in IVBA Technical-Planning Standards for Housing Complexes: analysis from Planning Research

Recepción: 06 Marzo 2020

Aprobación: 29 Junio 2020

Publicación: 30 Junio 2020

Recepción: 06 Marzo 2020

Aprobación: 29 Junio 2020

Publicación: 30 Junio 2020

\section{RESUMEN:}

Este ensayo describe cómo la adaptabilidad, una noción que recorre el corpus teórico de la arquitectura, se presenta en las Normas Técnicas para Proyecto de Conjuntos Habitacionales, con el objeto de ponderar los lineamientos que ofrece el Instituto de la Vivienda de la Provincia de Buenos Aires para su implementación. Se propone exponer cómo la normativa que se constituye como referencia técnica en la provincia de Buenos Aires, contribuye con la construcción de una representación conceptual en torno de la vivienda masiva. Partimos de la premisa de que existe una relación directa entre las características de la Vivienda Pública como proyecto -según las tres dimensiones que plantea la Investigación Proyectual: teórica, metodológica y técnica- y su capacidad para alcanzar niveles cualitativos satisfactorios, en el marco de lo que han establecido los Estándares Mínimos de Calidad para Viviendas de Interés Social. Palabras claves: adaptabilidad; proyecto; vivienda pública

Palabras ClaVE: adaptabilidad, proyecto, vivienda pública.

\section{Abstract:}

This essay describes how adaptability, a central concept in architectural theories, appears in IVBA Technical-Planning Standards for IVBA Housing Complexes, with de purpose of assess the Buenos Aires Housing Institute guidelines for it implementation. It is proposed to exhibit how referential technical regulation in Buenos Aires contributes with the construction of a conceptual representation of the massive housing. We start from the premise that exist a direct influence between the characteristics of Public Housing as project and its qualitative standards in Public Housing Minimal Qualitative Standards.

KEYWORDS: adaptability, planning, public housing.

\section{El IVBA y las Normas TÉcnicas en la DEFinición de UNA VIVIENDA ADAPTABLE}

En función de articular un diálogo entre normativa y proyecto, se propone describir brevemente los aspectos teórico-conceptuales de la adaptabilidad, en el marco de la vivienda de construcción masiva. En arquitectura, el término remite a la capacidad de cualquier edificación de admitir significativas modificaciones físicas y/o de uso (Bertuzzi, 2007) Como definición, contempla "flexibilidad" y "crecimiento", al referirse a transformaciones sobre las mismas condiciones de superficie o bien considerando la demanda incremental de metros cuadrados.

En los años 20, la idea de adaptabilidad se vio asociada con la vivienda masiva europea, y fue preconizada por los maestros modernos -y más tarde por la herencia y transculturación latinoamericana- en torno de la flexibilidad espacial. Hacia los años 60, en el marco socio-político del Estado de Bienestar, incursionan en el repertorio proyectual nociones tales como evolutividad y elasticidad vinculadas con el diseño tecnológico de la viviendas adaptables, y abrevando en las teorías de la Planificación Participativa. 
Podemos afirmar que el término aparece en los momentos más prolíficos de la producción teórica de la arquitectura, ligado en particular a una discusión sobre las características de la vivienda de cada época: un recurso estratégico para interpelar y re-definir sus aspectos más conservadores tanto en términos funcionales como de resolución constructiva. Del mismo modo y para la Vivienda Pública, se convierte en una estrategia destinada a optimizar su valor de uso.

La cuestión de la Vivienda Social encontró a finales de los años `60 y durante los años '70 un espacio protagónico en las políticas desarrollistas de los países del sur del continente americano. Se trata de un momento de propuestas de gran envergadura urbanística, en sintonía con las posibilidades tecnológicas y la confianza en ellas depositadas. Los conjuntos de media y alta densidad hicieron foco en los modos de agrupamiento de las unidades, en favor de articular espacios de uso colectivo en la interfase viviendaciudad. En las propuestas de baja densidad, la adaptabilidad se vio emparentada con la autoconstrucción y la adecuación de sus componentes a la capacidad ejecutiva de los destinatarios. El proyecto arquitectónico se adecua a los procesos autogestivos, y la Vivienda Social se comprendió como Producción Social del Hábitat. En la región, las dictaduras militares interrumpieron este incipiente período de indagaciones y experimentación de alternativas. El déficit habitacional siguió en ascenso, sin embargo las políticas económicas, condicionadas por el financiamiento de organismos internacionales, restringieron el volumen de inversiones destinadas a infraestructura y viviendas. Gradualmente declinaron las acciones orientadas a solucionar las problemáticas habitacionales de los sectores de menores recursos económicos y en este contexto, lo específicamente proyectual de la Vivienda de Interés Social, relativizó además su presencia en la discusión académica.

En los años '80, varios países europeos convocaron a concursos de arquitectura destinados a definir las viviendas que los nuevos modos de vida, comenzaban a demandar. El evento que marcó el punto de inicio de esta tendencia fue el primer Europan, convocado en 1988, cuyo lema "Le logement en question", sirvió como modelo para el concurso desarrollado al año siguiente en nueve países europeos. "Su enunciado afirmaba que mientras los modos de vida y las técnicas de construcción evolucionaban, la vivienda permanecia prisionera de modelos antiguos, marcos reglamentarios obsoletos y respuesta automáticas por parte del mercado inmobiliario"(Fernández Lorenzo, 2015:37)

En Argentina, la arquitectura de la Vivienda Pública enfrentó una democrática década del 80, sopesando el fracaso de los grandes conjuntos habitacionales. El gobierno orientó sus políticas al abordaje de la dimensión social y económica del problema de la vivienda. Aunque presentes la regularización dominial y fundamentalmente, la provisión extensiva de servicios urbanos, se fueron relegando las indagaciones proyectuales, que aún en la actualidad permanecen postergadas.

Por su parte, las políticas neoliberales de la década del 90 concretaron un puñado de acciones paliativas al déficit. Promoviendo la mercantilización de la Vivienda Social (Barreto, 2012; Fernández Wagner, 2015) se impulsó la formación de un mercado de capitales a través de la privatización parcial de los fondos de seguridad social para estimular créditos privados. Con este escenario, el Instituto de la Vivienda de la Provincia de Buenos Aires, creado en $1956^{1}$ por Decreto Ley No 469 , encuadró sus acciones en esta fase de descentralización de las políticas habitacionales. Éstas adquirieron una promoción significativa con el acuerdo con fuerza de Ley No 24130, firmado entre el gobierno nacional y los provinciales, que propuso la federalización del Fondo Nacional de la Vivienda (FONAVI). "El concepto de descentralización apunta a dinamizar de un modo prioritario los intereses propios de los grupos comunitarios a través de sus respectivos municipios, procurando de este modo, que sean los mismos destinatarios con la intermediación de sus legitimos representantes quienes propongan y gestionen las soluciones habitacionales acordes con el relevamiento de las necesidades locales que determinen las comunas". Por otro lado, en el año 1995 se creó por Ley No 24464 el Sistema Federal de la Vivienda (SFV), para promover el acceso a la vivienda. Entonces, el SFV reunía:

a) El FONAVI 
b) Los organismos provinciales y de la Ciudad Autónoma de Bs. As. encargados de aplicar la ley y administrar los recursos creados.

c) El Consejo Nacional de la Vivienda.

En el marco de esta nueva fase, el organismo presentó en 1992 un cuerpo de disposiciones denominado "Normas Técnicas para Proyectos de Conjuntos Habitacionales" (N.T.). El manual fue diseñado como "[...] un documento instrumental normativo válido como apoyatura técnica en lo referente al diseño urbanisticoarquitectónico, materiales y tecnologias tradicionales y elementos legales que formalicen la ejecución de viviendas y conjuntos de viviendas, orientadas a dar respuestas en el marco de los lineamientos estratégicos planteados ante la diversidad de problemas habitacionales que se manifiestan"2

En este sentido, las políticas de descentralización representaron para el IVBA una oportunidad para potenciar su rol como organismo rector en materia de vivienda. Recuperando la vasta experiencia técnicoproyectual adquirida desde su creación, el documento manifiesta la intención de posicionar al proyecto arquitectónico como parte de las soluciones de fondo. Aquí la adaptabilidad, que podría quedar circunscripta al ámbito académico, participa de una revisión de la vivienda, incluso desde los modos de habitar. ${ }^{3}$

\section{TeOría, METOdología y TÉCNICA DEL PROYECTO DE VIVIENDAS ADAPTABLES EN LAS N.T.}

La Investigación Proyectual ${ }^{4}$ reconoce que en términos de producción de conocimiento disciplinar, todo proyecto arquitectónico ofrece tres aspectos o dimensiones: una teoría, una metodología y una técnica. Considerando entonces la Vivienda Pública -los Conjuntos Habitacionales- como proyecto, y en el marco de lo establecido en el documento "Normas Técnicas para Proyectos de Conjuntos Habitacionales IVBA" respecto de adaptabilidad, nos proponemos caracterizar:

a. Respecto de la dimensión teórica: las definiciones y conceptos involucrados y consecuentemente indagar el posicionamiento teórico.

b. Respecto de la dimensión metodológica: las acciones: procedimientos e indicaciones que guían el ejercicio proyectual hacia la adaptabilidad.

c. Respecto de la dimensión técnica: las especificaciones de orden material, morfológico, dimensional y constructivo, es decir, los elementos de mayor concreción de los lineamientos técnico-proyectuales.

En las N.T. "flexibilidad" y "crecimiento" se presentan en el apartado 3.1.1, inciso c, relacionado con el Diseño de la Vivienda. Coexisten en el cuerpo del documento con la recreación y variación tipológica, las expansiones, el criterio de racionalidad general y las previsiones especiales vinculadas con la accesibilidad. Reconoce previamente dos tipos de espacios, que reciben la denominación de "unidades habitacionales": viviendas y locales. Las especificaciones que a ambos refieren, reconocen una categorización según la escala o complejidad que presenta su abordaje: de la célula en sí mismo, en relación a su agrupamiento y respecto de su posición en la propuesta de conjunto. "Trátese de las determinaciones proyectuales y técnicas de la unidad habitacional en si misma, en relación al conjunto, a las relaciones comparativas entre otras y a su resolución particular"5

En lo específico en texto señala: "La unidad deberá presentar criterios probados deflexibilidady crecimiento"

Para "flexibilidad" señala: "La forma y proporción racional de cada ámbito, la intercomunicación fluida entre los mismos, la posibilidad de divisiones virtuales entre si y su ubicación, acceder al cambio de destino y la opción en el armado del amoblamiento. Esto dependerá de eliminar al máximo las circulaciones internas. Deberán cuidarse las minimas condiciones de iluminación y ventilación al preverse las divisiones internas" 7

Y para "crecimiento", el texto expresa: "En el caso de viviendas individuales en lotes propios, deberia darse el crecimiento de los dormitorios hacia el fondo de lote, evitando el cambio de imagen desorganizado de conjunto. En el casos de vivienda colectiva, se aconseja atender como crecimiento la disposición y subdivisión interna $y$ sus alternativas en viviendas más generosas, donde el crecimiento general en $m^{2}$ sea minimo y previsto 
tecnológicamente" Y luego continúa: "Se corroborará todo criterio de crecimiento con el detalle y previsión tecnológica que lo avale. Por lo tanto el crecimiento se efectuará sin demolición. Se permitirá la remoción y/o traslado de paneles, trabas de ladrillos preparadas para proseguirlas, traslado o apertura de carpinterías móviles fijas." $^{\prime}$

\section{ANÁLISIS DESDE LA I.P.: CONCEPTOS, PROCEDIMIENTOS Y SUBSISTEMAS9}

En primer lugar podemos señalar que adaptabilidad, en tanto "Flexibilidad" y "Crecimiento" resulta una de las "determinaciones proyectuales" que las N.T. presentan en relación con la Unidad Habitacional, y dentro del apartado "Diseño". Debemos señalar que los aspectos proyectuales se exponen por separado de aquellos "técnicos", aun cuando se presenta una definición de vivienda que debe promover "[...] una relación indisoluble entre diseño, proyecto, racionalidad y economia". Cabe destacar además que diseño resulta entonces diferente de proyecto, en el marco de una aproximación conceptual en la que el primero remite a los componentes o subsistemas de la unidad y el segundo aparece como sinónimo de resolución integral o totalidad. "Especificamente se refiere a los problemas del diseño de las partes constitutivas"

Por otro lado, señalar que la iniciativa pondera el rol de la disciplina arquitectónica en la definición de lineamientos o parámetro que permiten definir los aspectos cualitativos de la Vivienda Pública, en los términos en los que se trasciende la experiencia estrictamente técnica y se da lugar a criterios técnicoproyectuales. En este sentido, involucrar los valores de proyecto en el abordaje significa entonces asignarle a la arquitectura un destacable campo de acción. Sin embargo, las N.T. todavía no posicional al "Diseño" como premisa rectora de la "Habitabilidad" de la vivienda. Para esta condición -apartado 4º sólo "[... se destaca la importancia de la envolvente de la vivienda en la obtención de la habitabilidad de la misma".

Por último destacar el valora de la iniciativa por su aporte:

- teórico-referencial: ofrece un soporte conceptual como condición de partida

- instrumental-procedimental: operacionaliza acciones técnicas y de diseño.

- técnico-normativo: garantiza Estándares Mínimos de Calidad en la V. Social ${ }^{15}$

El apartado que expone lo específico respecto de "Flexibilidad" y "Crecimiento" comienza por señalar que "La unidad deberá presentar criterios probados de flexibilidad y crecimiento". Nos preguntamos entonces si la validez de los recursos a los que se hace mención depende de su reconocimiento en el arsenal de propuestas que constituyen los antecedentes proyectuales del IVBA. Si esto fuera así -y aun cuando no se explicitan- el hecho de validar estrategias desde la propia práctica remite a un posicionamiento próximo al que propone la Investigación Proyectual, en los términos en que la disciplina corrobora con cada proyecto una premisa, y que esto, al modo de una hipótesis contrafáctica ${ }^{10}$, constituye la producción específica del conocimiento.

En adelante, el documento presenta los conceptos forma, proporción, intercomunicación, fluidez, virtualidad y cambio de destino. Todas estas nociones resultan constantes en el corpus teórico de la arquitectura, y podríamos afirmar que las últimas han interpelado la constitución tipológica del espacio doméstico desde el proyecto moderno hasta nuestros días. Sin embargo, estas variables aparecen para "flexibilidad" asociadas únicamente con una condición de partida: “[...] esto dependerá de eliminar al máximo las circulaciones internas” El carácter reduccionista de la estrategia, que remite a las teorías proyectuales en clave cientificista de la optimización del sistema de movimiento -Alexander Klein, entre otros ${ }^{11}$-no se corresponde aparentemente con las premisas generales

Al finalizar, el último apartado señala que “[...] deberán cuidarse las mínimas condiciones de iluminación y ventilación al preverse subdivisiones internas"12. Nos resulta singular esta afirmación al menos por tres apectos -teoría, metodología y técnica: 
- promueve la conceptualización -y proyectación- de una planta compartimentable ${ }^{13,}$ y consecuentemente desestima la concepción tradicional a partir de una serie de ámbitos estancos totalmente definidos de antemano.

- promueve la divisibilidad como estrategia proyectual hacia la adaptabilidad (Bertuzzi, 2007) y consecuentemente interpela la vivienda que queda reducida a su mínimo dimensional.

- promueve la articulación de dos subsistemas de la unidad edilicia -envolvente y particiones- en pos de garantizar márgenes de adaptabilidad. Esto tensiona incluso los criterios compositivos de las fachadas: es ahora la variable temporal la que podría determinar la posición del aventanamiento atendiendo a usos a futuro. Los recursos proyectuales (Bertuzzi, 2007) como elementos de mayor concreción en pos de la adaptabilidad, constituyen la dimensión técnica de la premisa, y serán los encargados de garantizar las deseables condiciones en el tratamiento de la luz y la ventilación.

Por otro lado, respecto de "crecimiento" se presenta una diferenciación tipológica: el aumento de superficie adicional resulta potestad exclusiva de la vivienda individual en lote propio. Para el caso de agrupaciones colectivas, se recomienda "[...] entender como crecimiento la disposición y subdivisión interna y sus alternativas en viviendas más generosas, donde el crecimiento general en $\mathrm{m}^{2}$ sea minimo y previsto tecnológicamente." Sin embargo, el crecimiento a partir del recurso morfológico-dimensional "terraza exterior edificable", presente en numerosos ejemplos de vivienda individual en lote propio, ¿no pondría en crisis las anteriores afirmaciones?

A pesar de la asignación teórica de monofuncionalidad a la vivienda, y de la tipificación excesivamente simplificada de las actividades -comer, dormir, estar, etc.- las estrategias que rescatamos como dimensión metodológica -en particular las relacionadas con "crecimiento" tienden a revisar las resoluciones constructivas como materializaciones a perpetuidad. En este sentido, el recurso proyectual predilecto resulta la estructura puntual independiente. "[...] el crecimiento se efectuará sin demolición. Se permitirá la remoción y/ o traslado de paneles, trabas de ladrillos preparadas para proseguirlas, traslado o apertura de carpinterias móviles fijas" $^{14}$

En términos de dimensión técnica, la envolvente y las particiones resultan los componentes protagonistas e incluso su consideración conjunta resulta para las N.T. un ítem deseable. Sin embargo, para concebir los conjuntos habitacionales como proyecto, al menos desde el marco que propone la Investigación Proyectual, las N.T. deberían establecer precisiones respecto de sus aspectos constitutivos: teoría, metodología y técnica. Estas especificaciones no sólo garantizarían adecuados niveles de adaptabilidad, sino respecto del resto de las variables enunciadas en el apartado "Diseño", tanto de la unidad como del conjunto. Asimismo, el abordaje de las tres dimensiones abre según cada una de ellas, especificidades susceptibles de continuidad, con miras a la producción de conocimiento: innovaciones técnicas, modalidades tipológicas experimentales, procedimientos y técnicas del hacer proyectual. Se valora entonces que en las N.T. se proponga establecer como proyectos a los conjuntos habitacionales, como condición de partida.

\section{Bibliografía}

Barreto, M.A. (2012) Cambios y continuidades en la politica de vivienda argentina (2003-2007). Bogotá: Pontificia Javeriana.

Bertuzzi, H. (2007) Adaptabilidad es más. Estrategias y recursos para el diseño de viviendas adaptables. Mar del Plata: Eudem.

Fernández Lorenzo, P. (2015) Hacia una vivienda abierta concebida como si el habitante importara. Buenos Aires: Diseño. 
Fernández Wagner, R. (2015) El sistema de la vivienda pública en Argentina. Revisión desde la perspectiva de los regímenes de vivienda. En M.A. Barreto y M. Lentini (comps.) Hacia una politica integral de hábitat. Aportes para un observatorio de politica habitacional en Argentina. (pp.29-96). Buenos Aires: Café de las ciudades.

Fiscarelli, D. (2016) Adaptabilidad y vivienda de producción estatal: estrategias y recursos proyectuales. SubPrograma de Urbanización de Villas y Asentamientos Precarios 2005-2009.(Tesis Doctoral). http://sedici.unlp.edu.ar/handl e/10915/58682

Sarquis, J. (2005) Arquitectura y modos de habitar. Buenos Aires: Nobuko.

Sarquis, J. (2007) Itinerarios del proyecto. La Investigación Proyectual como forma de conocimiento en Arquitectura. Buenos Aires: Nobuko

\section{Notas}

* Docente Investigador Categoría V UNLP. Dr. en Arquitectura FAU UNLP. Especialista en Investigación Proyectual orientación Vivienda FADU UBA. Actualmente becario posdoctoral del Consejo Nacional de Investigaciones Científicas y Técnicas CONICET. Como línea de investigación desarrolla el tema Vivienda Pública y proyecto arquitectónico, con énfasis en la articulación entre aspectos sociales, la tecnología y las posibilidades funcionales que demandan el habitar contemporáneo en las ciudades. Ha dictado seminarios y talleres exponiendo la problemática del hábitat urbano en relación a los cambios que han sufrido las constituciones familiares durante las últimas décadas en Argentina.

1 El IVBA en la actualidad sostiene como objetivos brindar asesoramiento técnico-proyectual a los entes provinciales y municipales promotores de la Vivienda Pública, y además impulsar a la iniciativa privada a que adquiera y construya unidades.

2 IVBA. (1992) Normas Técnicas para Proyectos de Conjuntos Habitacionales. Pág. 2

3 "Durante años, la destrucción de la trama urbana por medio de los macroconjuntos hacían y hacen notar al IVBA como generador de ámbitos más proclives a producir desviaciones sociales que a favorecer la vida en comunidad. Esta situación se revierte asumiendo el respeto por la generación urbana, las leyes de la ciudad, el relevamiento y la actitud crítica ante los tipos urbanos (plazas, calles, líneas de fachada, etc.) y tipos de viviendas (reflejando los genuinos modos de vida) como temas fundamentales de las soluciones de fondo". IVBA. (1992) Normas Técnicas para Proyectos de Conjuntos Habitacionales. Pág. 6.

4 "La Investigación Proyectual es una teoria que propone realizar proyectos con el objeto de obtener conocimientos disciplinares. Se caracteriza por encausar el procedimiento proyectual atendiendo a aspectos que no atienden los proyectos profesionales o formativos tradicionales. Fijar un punto fundante en dos atores que, en nuestra teoria de la arquitectura, son protagonistas fundamentales: el proyectista como creador imprescindible de la forma arquitectónica y la sociedad encarnada en el usuario como receptor re-creador de esa forma, mediante un habitar que trasciende el mero uso y va hacia un habitar creativo" En: Sarquis, J. (2007) "Itinerarios del proyecto. La Investigación Proyectual como forma de conocimiento en Arquitectura". Buenos Aires: Ed. Nobuko.

5 IVBA. (1992) Normas Técnicas para Proyectos de Conjuntos Habitacionales. Pág. 15

6 IVBA. (1992) Normas Técnicas para Proyectos de Conjuntos Habitacionales. Pág. 16

7 IVBA. (1992) Normas Técnicas para Proyectos de Conjuntos Habitacionales. Pág. 16

8 IVBA. (1992) Normas Técnicas para Proyectos de Conjuntos Habitacionales. Pág. 16

9 "Las siguientes normas reflejan el marco teórico que asegure un minimo de calidad en los proyectos propiciados por el Instituto de la Vivienda de la Provincia de Buenos Aires a través de Entidades Públicas y/o privadas y extensivos a los elaborados por el Instituto" IVBA. (1992) Normas Técnicas para Proyectos de Conjuntos Habitacionales. Pág. 6

10 Ver: Fernández, R. (2013) Inteligencia Proyectual. Un manual de investigación en arquitectura. Buenos Aires: Teseo.

11 Dentro de esta línea argumental podemos posicionar los primeros trabajos de Alexander Klein, quien en 1928 -y con el patrocinio económico del estado alemán- presentó su “Casa Funcional para una vida sin fricción”. En esta propuesta, cuya exposición al público se valía del estudio comparativo de una vivienda típica del Siglo XIX, se estudiaba en detalle el sistema de movimiento, en tanto optimización de las circulaciones necesarias para pasar de un ámbito a otro, con el objeto de evitar que se produjeran cruces inoportunos entre los miembros de la Unidad Doméstica. Ver: Klein, A. (1980) -reimpresión- “La vivienda minima. 1906-1957” Barcelona: Ed. G.G.

12 IVBA. (1992) Normas Técnicas para Proyectos de Conjuntos Habitacionales. Pág. 15

13 Planta compartimentable resulta una de las modalidades tipológicas que han sido estudiadas en la Tesis Doctoral "Adaptabilidad y vivienda de producción estatal: estrategias y recursos proyectuales. SubPrograma de Urbanización 
de Villas y Asentamientos Precarios. 2005-2009”. Entre otras variantes encontramos vivienda expansible, vivienda progresiva, vivienda divisible-unificable y vivienda completable.

14 IVBA. (1992) Normas Técnicas para Proyectos de Conjuntos Habitacionales. Pág. 15

15 SubSecretaría de Desarrollo Urbano y Vivienda. Secretaria de Obras Públicas. Ministerio de Planificación Federal, Inversión Pública y Servicios. (2006) Estándares Minimos de Calidad para Viviendas de Interés Social

\section{BY-NC-SA}

